\title{
FINITE EXPONENTIAL SERIES AND NEWMAN POLYNOMIALS
}

\author{
BART GODDARD
}

(Communicated by Clifford J. Earle, Jr.)

\begin{abstract}
A Newman polynomial is a sum of powers of $z$, with constant term 1. The Newman polynomial of four terms whose minimum modulus on the unit circle is as large as possible is found by examining the expression

$$
f(4)=\sup _{x_{1}<\cdots<x_{4}} \inf _{\alpha \in \mathfrak{R}}\left|\sum_{j=1}^{4} e^{i x_{j} \alpha}\right|
$$

and determining an extremal system $\left(x_{1}, \ldots, x_{4}\right)$ using a technique that reduces the problem to a finite search.
\end{abstract}

\section{INTRODUCTION}

Let $P(z)=\sum_{j=1}^{n} a_{j} z^{r_{j}}$ be a complex polynomial. Erdös [1] and Littlewood [2] asked several questions concerning the minimum modulus of $P(z)$ on the unit circle, under various restrictions of the coefficients $a_{j}$, e.g., $\left|a_{j}\right|=1$ for $j=1,2, \ldots, n$. If we insist that $a_{j}=1$ for $j=2, \ldots, n$ and $r_{1}=0$, then $P(z)$ is a Newman polynomial, as defined by Campbell, Ferguson, and Forcade [3]. Many other authors have investigated the minimum modulus of Newman polynomials, most notably Smyth [4] and Boyd [5].

Rudolfer and Hayman [7] ask for information about

$$
f(n)=\sup _{x_{1}<x_{2}<\cdots<x_{n}} \inf _{\alpha \in \mathfrak{R}}\left|\sum_{j=1}^{n} e^{i \alpha x_{j}}\right| .
$$

If $x_{1}=r_{1}, x_{2}=r_{2}, \ldots, x_{n}=r_{n}$ are natural numbers, we have

$$
f(n)=\sup _{r_{1}<r_{2}<\cdots<r_{n}} \min _{|z|=1}|P(z)| .
$$

The purpose of this paper is to calculate $f(4)$ explicitly and, in the process, discover some examples of Newman polynomials with few terms, but large minimum modulus. $f(2)$ is trivially 0 , and $f(3)$ is calculated in [3], being

Received by the editors December 11, 1990 and, in revised form, March 14, 1991.

1991 Mathematics Subject Classification. Primary 11L03; Secondary 30C10.

Key words and phrases. Newman polynomials, exponential sums. 
attained for the Newman polynomial $1+z^{2}+z^{3}$. We shall prove here that $f(4)$ is attained for $1+z^{2}+z^{3}+z^{4}$.

\section{Preliminaries}

For $n$ a natural number, we define $F_{n}: \mathbb{R}^{n} \rightarrow \mathbb{R}$ via

$$
F_{n}\left(x_{1}, \ldots, x_{n}\right)=\left|\sum_{j=1}^{n} e^{i x_{j}}\right|^{2}
$$

Then we have $f(n)=\sup _{x_{1}<\cdots<x_{n}} \inf _{\alpha \in \mathfrak{R}} F_{n}\left(x_{1} \alpha, \ldots, x_{n} \alpha\right)^{1 / 2}$. It is easy to show that

$$
F_{n}\left(x_{1}, \ldots, x_{n}\right)=n+2 \sum_{j<k} \cos \left(x_{k}-x_{j}\right),
$$

and from this, that

$$
F_{n}\left(x_{1}, x_{2}, \ldots, x_{n}\right)=F_{n}\left(x_{1}, x_{n}+x_{1}-x_{n-1}, \ldots, x_{n}+x_{1}-x_{2}, x_{n}\right) .
$$

The simplification

$$
f(n)=\sup _{0=A_{1}<A_{2}<\cdots<A_{n}} \inf _{\alpha \in \mathfrak{R}} F_{n}\left(0, A_{2} \alpha, \ldots, A_{n} \alpha\right)^{1 / 2}
$$

where $0=A_{1}<A_{2}<\cdots<A_{n}$ are nonnegative integers and $\operatorname{gcd}\left(A_{2}, A_{3}, \ldots, A_{n}\right)$ $=1$, is given as Theorem 1 of [3], or we may proceed as follows: It suffices to show that for every $n$-tuple $\left(x_{1}, \ldots, x_{n}\right) \in \mathbb{R}^{n}$ and $\varepsilon>0$ there is an $n$-tuple $\left(r_{1}, \ldots, r_{n}\right) \in \mathbb{Q}^{n}$ such that

$$
\inf _{\alpha \in \mathfrak{R}}\left|\sum_{j=1}^{n} e^{i \alpha x_{j}}\right| \leq \inf _{\alpha \in \mathfrak{R}}\left|\sum_{j=1}^{n} e^{i \alpha r_{j}}\right|+\varepsilon .
$$

If each $x_{j} \in \mathbb{Q}$, we are done. Otherwise, by the simultaneous rational approximation theorem (Hardy and Wright [8, p. 170]) there are infinitely many solutions to the system of inequalities

$$
\left|x_{j}-\frac{p_{j}}{q}\right|<\frac{1}{q^{(1+1 / n)}}, \quad j=1,2, \ldots, n .
$$

Further, the function $h(x)=e^{i x}$ is continuous and periodic, hence uniformly continuous over $\mathbb{R}$, so there exists a $\delta>0$ such that $\left|e^{i x}-e^{i y}\right|<\varepsilon / n$ whenever $|x-y|<\delta$. Let $\left[p_{1} / q, \ldots, p_{n} / q\right]$ be a solution to the inequalities with $q>$ $[2 \pi / \delta]^{n}$. Then $q^{1 / n}>2 \pi / \delta$, and hence $\delta>2 \pi / q^{1 / n}$. Then the function of $\alpha, g(\alpha)=\left|\sum_{j=1}^{n} e^{i \alpha p_{j} / q}\right|$ has period $2 \pi q$. Now for $0 \leq \alpha \leq 2 \pi q$ we have

$$
\left|\sum_{j=1}^{n} e^{i \alpha x_{j}}-\sum_{j=1}^{n} e^{i \alpha p_{j} / q}\right| \leq \sum_{j=1}^{n}\left|e^{i \alpha x_{j}}-e^{i \alpha p_{j} / q}\right| \leq \sum_{j=1}^{n} \frac{\varepsilon}{n}=\varepsilon
$$

since $\left|\alpha x_{j}-\alpha p_{j} / q\right|=\alpha\left|x_{j}-p_{j} / q\right|<2 \pi q / q^{(1+1 / n)}=2 \pi / q^{1 / n}<\delta$.

So,

$$
\inf _{\alpha \in[0,2 \pi q]}\left|\sum_{j=1}^{n} e^{i \alpha x_{j}}\right| \leq \inf _{a \in[0,2 \pi q]}\left|\sum_{j=1}^{n} e^{i \alpha p_{j} / q}\right|+\varepsilon .
$$


Then, since we are taking the infimum over a smaller set, we have

$$
\begin{aligned}
\inf _{\alpha \in \mathfrak{R}}\left|\sum_{j=1}^{n} e^{i \alpha x_{j}}\right| & \leq \inf _{a \in[0,2 \pi q]}\left|\sum_{j=1}^{n} e^{i \alpha x_{j}}\right| \\
& \leq \inf _{a \in[0,2 \pi q]}\left|\sum_{j=1}^{n} e^{i \alpha p_{j} / q}\right|+\varepsilon=\inf _{\alpha \in \mathfrak{R}}\left|\sum_{j=1}^{n} e^{i \alpha p_{j} / q}\right|+\varepsilon
\end{aligned}
$$

since $g(\alpha)$ has period $2 \pi q$. Consequently, the following variation of (3) is considered:

(4)

$$
F_{n}\left(0, A_{2}, A_{3}, \ldots, A_{n}\right)=F_{n}\left(0, A_{n}-A_{n-1}, A_{n}-A_{n-2}, \ldots A_{n}-A_{2}, A_{n}\right) .
$$

\section{LEMMAS}

We will need the following lemmas. Recall from (1) and (2) that

$$
F_{4}\left(x_{1}, x_{2}, x_{3}, x_{4}\right)=\left|\sum_{j=1}^{4} e^{i x_{j}}\right|^{2}=4+2 \sum_{j<k} \cos \left(x_{k}-x_{j}\right)
$$

Lemma 1. Given distinct integers $\left(x_{1}, x_{2}, x_{3}, x_{4}\right)$, there is a zero $\left(z_{1}, z_{2}, z_{3}\right.$, $\left.z_{4}\right)$ of $F_{4}$ and a $t_{0} \in \mathbb{R}$ such that

(i) $x_{2} t_{0}=z_{2} ; \quad x_{3} t_{0}=z_{3} ; \quad x_{4} t_{0}=z_{4}$;

(ii) $\left|z_{1}-x_{1} t_{0}\right| \leq \pi \operatorname{gcd}\left(x_{4}-x_{3}, x_{2}-x_{1}\right) /\left(\left|x_{4}-x_{3}\right|\right)$; and

(iii) $\left(z_{4}-z_{3}\right)$ and $\left(z_{2}-z_{1}\right)$ are odd multiples of $\pi$.

Proof. Let $d=\operatorname{gcd}\left(x_{4}-x_{3}, x_{2}-x_{1}\right)$. Consider the linear Diophantine equation in $l$ and $k$,

$$
2 l\left(x_{2}-x_{1}\right)+2 k\left(x_{4}-x_{3}\right)=\left(x_{1}-x_{2}\right)+\left(x_{3}-x_{4}\right)+\beta d
$$

where $\beta=0$ or 1 is chosen so that the right-hand side is an even multiple of $d$. With $\beta$ so chosen, (5) is solvable. Let $l=l_{0}$ and $k=k_{0}$ be a solution. Let $t_{0}=\left(2 l_{0}+1\right) \pi /\left(x_{4}-x_{3}\right), z_{1}=t_{0} x_{2}+\left(2 k_{0}+1\right) \pi, z_{2}=x_{2} t_{0}, z_{3}=x_{3} t_{0}$, and $z_{4}=x_{4} t_{0}$. Then it is easy to check that $\left(z_{4}-z_{3}\right)=\left(2 l_{0}+1\right) \pi$ and $\left(z_{2}-z_{1}\right)=-\left(2 k_{0}+1\right) \pi$. It remains to show (ii) is satisfied:

$$
\begin{aligned}
\left|z_{1}-x_{1} t_{0}\right| & =\left|t_{0} x_{2}+\left(2 k_{0}+1\right) \pi-x_{1} t_{0}\right| \\
& =\left|t_{0}\left(x_{2}-x_{1}\right)+\left(2 k_{0}+1\right) \pi\right| \\
& =\left|\frac{\left(2 l_{0}+1\right) \pi}{x_{4}-x_{3}}\left(x_{2}-x_{1}\right)+\left(2 k_{0}+1\right) \pi\right| \\
& =\frac{\pi}{\left|x_{4}-x_{3}\right|}\left|\left(2 l_{0}+1\right)\left(x_{2}-x_{1}\right)+\left(2 k_{0}+1\right)\left(x_{4}-x_{3}\right)\right| \\
& =\frac{\pi}{\left|x_{4}-x_{3}\right|}|\beta d| \leq \frac{\pi d}{\left|x_{4}-x_{3}\right|},
\end{aligned}
$$

which completes the proof.

Lemma 2. Let $w$ be a positive real number. Let $A_{1}, A_{2}, A_{3}$ be distinct natural numbers such that $\operatorname{gcd}\left(A_{1}, A_{2}, A_{3}\right)=1$ and $w\left(\operatorname{gcd}\left(A_{1}, A_{2}-A_{3}\right)\right) \leq A_{1}$. Then

$$
\inf _{\alpha \in \mathfrak{R}} F_{4}\left(0, A_{1} \alpha, A_{2} \alpha, A_{3} \alpha\right)<(\pi / w)^{2} \text {. }
$$


Proof. Let $d=\left(A_{1}, A_{2}-A_{3}\right)$. Then we have $d / A_{1} \leq 1 / w$. For the point $\left(0, A_{1}, A_{2}, A_{3}\right)$ in $\mathbb{R}^{4}$, Lemma 1 gives a zero $\left(z_{1}, z_{2}, z_{3}, z_{4}\right) \in \mathbb{R}^{4}$ and an $\alpha_{0} \in \mathbb{R}$ such that $0 \alpha_{0}=z_{1}, A_{1} \alpha_{0}=z_{2}, A_{2} \alpha_{0}=z_{3}$, and

$$
\left|A_{3} \alpha_{0}-z_{4}\right|<\frac{\pi \cdot \operatorname{gcd}\left(A_{1}, A_{3}-A_{2}\right)}{A_{1}}=\frac{\pi d}{A_{1}} \leq \frac{\pi}{w} .
$$

Further, $z_{2}=\left(z_{2}-z_{1}\right)$ and $\left(z_{4}-z_{3}\right)$ are odd multiples of $\pi$, say $z_{2}=$ $\left(z_{2}-z_{1}\right)=(2 k+1) \pi$ and $\left(z_{4}-z_{3}\right)=(2 l+1) \pi$. Let $\gamma=A_{3} \alpha_{0}-z_{4}$. Now we compute

$$
\begin{aligned}
\inf _{a \in \mathfrak{R}} & F_{4}\left(0, A_{1} \alpha, A_{2} \alpha, A_{3} \alpha\right) \leq F_{4}\left(0, A_{1} \alpha_{0}, A_{2} \alpha_{0}, A_{3} \alpha_{0}\right) \\
& =\left|1+e^{i z_{2}}+e^{i z_{3}}+e^{i A_{3} \alpha_{0}}\right|^{2} \\
& =\left|1+e^{i(2 k+1) \pi}+e^{i z_{4}}\left(e^{i\left(z_{3}-z_{4}\right)}+e^{i\left(A_{3} \alpha-z_{4}\right)}\right)\right|^{2} \\
& =\left|1-1+e^{i z_{4}}\left(e^{-i(2 l+1) \pi}+e^{i \gamma}\right)\right|^{2} \\
& =\left|e^{i z_{4}}\right|^{2}\left|-1+e^{i \gamma}\right|^{2}=1 \cdot\left|e^{i \gamma / 2}-e^{-i \gamma / 2}\right|^{2} \\
& =4 \sin ^{2}\left(\frac{\gamma}{2}\right) \leq 4\left(\frac{\gamma}{2}\right)^{2}=\gamma^{2}<\left(\frac{\pi}{w}\right)^{2}, \quad \text { as desired. }
\end{aligned}
$$

Lemma 3. Let $(a, b, c)$ be a triple of natural numbers such that
(i) $a<b<c$,
(ii) $\operatorname{gcd}(a, b, c)=1$,
(iii) $c-a<b$,
(iv) $\operatorname{gcd}(a, c-b)>a / 4.18$,
(v) $\operatorname{gcd}(b, c-a)>b / 4.18$,
(vi) $\operatorname{gcd}(c, b-a)>c / 4.18$.

Then $(a, b, c)=(2,3,4)$ or $(4,9,10)$.

Proof. Since $\operatorname{gcd}(c, b-a)$ is a divisor of $c, \operatorname{gcd}(c, b-a) / c$ is a rational number of the form $1 / m$ where $m$ is a natural number. Then $1 / m>1 / 4.18$ from (vi). Whence $m<4.18$. Since $b-a<c, \operatorname{gcd}(c, b-a)<c$, so $m \neq 1$. Therefore, the possible values of $m$ are 2,3 , and 4 .

If $m=2$ then $b-a=c / 2$, hence $2 b-2 a=c$.

If $m=3$ then $b-a=c / 3$ or $2 c / 3$, so $3 b-3 a=c$ or $3 b-3 a=2 c$.

If $m=4$ then $b-a=c / 4$ or $3 c / 4$, so $4 b-4 a=c$ or $4 b-4 a=3 c$.

So $(a, b, c)$ must satisfy one of the five Diophantine equations:

$$
\begin{gathered}
2 b-2 a=c, \quad 3 b-3 a=c, \quad 3 b-3 a=2 c, \\
4 b-4 a=c, \quad 4 b-4 a=3 c .
\end{gathered}
$$

Similarly, using inequalities (v) and (iii), we have that $(a, b, c)$ must satisfy one of the five Diophantine equations:

$$
\begin{gathered}
2 c-2 a=b, \quad 3 c-3 a=b, \quad 3 c-3 a=2 b, \\
4 c-4 a=b, \quad 4 c-4 a=3 b .
\end{gathered}
$$

First suppose $k(b-a)=l c$ and $j(c-a)=i b$ for integers $k, l, j$, and $i$; that is, $(a, b, c)$ satisfy one of the first five and one of the second five equations 
above. Then solving for $b$ and $c$ in terms of $a$ yields

$$
\begin{gathered}
c=\left[\frac{k j+k i}{k j-l i}\right] a, \\
b=\left[\frac{k j+l i}{k j-l i}\right] a .
\end{gathered}
$$

Define $t$ by $a=(k j-l i) t$. Then from (6) and (7) $b=\frac{(k j+l j)}{(k j-l i)}(k j-l i) t=(k j+l i) t$ and $c=\frac{(k j+k i)}{(k j-l i)}(k j-l i) t=(k j+k i) t$.

Then $t$ is rational, say $t=p / q$ with $p, q$ relatively prime integers. Then $p \mid a, b$, and $c$, whence $p=1$. So $t=1 / q$ for some natural number $q$. Then $q \mid(k j-l i),(k j+l j)$, and $(k j+k i)$. Since $\operatorname{gcd}(a, b, c)=1$, we must have $q=\operatorname{gcd}((k j-l i),(k j+l j),(k j+k i))$. Thus there is only one solution to the Diophantine system

$$
\left\{\begin{array}{l}
k(b-a)=l c \\
j(c-a)=i b
\end{array}\right.
$$

with $(a, b, c)=1$. Also, since $b<c$, we have $(k j+l j) t<(k j+k i) t$. So $l j<k i$. There are 25 possibilities for the tuple $(k, l, j, i)$, corresponding to the 25 possible Diophantine systems. The following table lists those tuples with $l j<k i$, along with the value of $q$ and the corresponding solution $(a, b, c)$.

Table I

\begin{tabular}{cccccc}
$k, l, j, i$ & $k j-l i$ & $k j+l j$ & $k j+k i$ & $q$ & $(a, b, c)$ \\
\hline $2,1,3,2$ & 4 & 9 & 10 & 1 & $(4,9,10)$ \\
$2,1,4,3$ & 5 & 12 & 14 & 1 & $(5,12,14)$ \\
$3,1,2,1$ & 5 & 8 & 9 & 1 & $(5,8,9)$ \\
$3,1,3,2$ & 7 & 12 & 15 & 1 & $(7,12,15)$ \\
$3,1,4,3$ & 9 & 16 & 21 & 1 & $(9,16,21)$ \\
$3,2,4,3$ & 6 & 20 & 21 & 1 & $(6,20,21)$ \\
$4,1,2,1$ & 7 & 10 & 12 & 1 & $(7,10,12)$ \\
$4,1,3,1$ & 11 & 15 & 16 & 1 & $(11,15,16)$ \\
$4,1,3,2$ & 10 & 15 & 20 & 5 & $(2,3,4)$ \\
$4,1,4,3$ & 13 & 20 & 28 & 1 & $(13,20,28)$
\end{tabular}

Now it remains to eliminate most of the triples $(a, b, c)$ in Table I, by showing that they violate one of the inequalities (iv) $-(\mathrm{vi})$. Now $c-a<b$, so $c-b<a$; whence $a \nmid(c-b)$, so if $a$ is a prime bigger than 4 , we have

$$
\frac{\operatorname{gcd}(a, c-b)}{a}=\frac{1}{a} \leq \frac{1}{5}<\frac{1}{4.18},
$$


so the triples $(5,12,14),(5,8,9),(7,12,15),(7,10,12),(11,15,16)$, and $(13,20,28)$ violate inequality (iv). This leaves only $(4,9,10),(9,16$, $21),(6,20,21)$, and $(2,3,4)$. But

$$
\frac{\operatorname{gcd}(9,21-16)}{9}=\frac{\operatorname{gcd}(9,5)}{9}=\frac{1}{9}<\frac{1}{4.18}
$$

and

$$
\frac{\operatorname{gcd}(6,21-20)}{6}=\frac{1}{6}<\frac{1}{4.18},
$$

which violate (iv). This leaves $(4,9,10)$ and $(2,3,4)$ as claimed.

Lemma 4. $\inf _{\alpha \in \mathfrak{R}} F_{4}(0,2 \alpha, 3 \alpha, 4 \alpha)=0.566 \ldots$.

Proof. From (3) and the Chebyshev polynomials,

$$
\begin{aligned}
F_{4}(0,2 \alpha, 3 \alpha, 4 \alpha) & =4+2(\cos 2 \alpha+\cos 3 \alpha+\cos 4 \alpha+\cos \alpha+\cos 2 \alpha+\cos \alpha) \\
& =16 \cos ^{4} \alpha+8 \cos ^{3} \alpha-8 \cos ^{2} \alpha-2 \cos \alpha+2 .
\end{aligned}
$$

Then

$$
\inf _{\alpha \in \mathfrak{R}} F_{4}(0,2 \alpha, 3 \alpha, 4 \alpha)=\min _{-1 \leq x \leq 1}\left(16 x^{4}+8 x^{3}-8 x^{2}-2 x+2\right) .
$$

It is a simple calculus exercise to show this last expression is equal to $0.566 \ldots$ as desired.

Note that this is equivalent to saying

$$
\min _{|z|=1}\left|1+z^{2}+z^{3}+z^{4}\right|=(0.566 \ldots)^{1 / 2}=0.7524 \ldots,
$$

which appears in Table 1 of [5]. Thus we have a Newman polynomial of only four terms, with relatively large minimum modulus on the unit circle. The next theorem shows this result is best possible.

\section{MAIN Result}

Theorem 1. $f(4)=0.7524 \ldots$.

Proof. Let $A_{1}<A_{2}<A_{3}$ be distinct positive integers with $\left(A_{1}, A_{2}, A_{3}\right)=1$. From the functional relationship (3), we have

$$
F_{4}\left(0, A_{1} \alpha, A_{2} \alpha, A_{3} \alpha\right)=F_{4}\left(0,\left(A_{3}-A_{2}\right) \alpha,\left(A_{3}-A_{1}\right) \alpha, A_{3} \alpha\right),
$$

so if $A_{3}-A_{1}>A_{2}$ let $A_{1}^{\prime}=A_{3}-A_{2}, A_{2}^{\prime}=A_{3}-A_{1}$, and $A_{3}^{\prime}=A_{3}$. Then $A_{3}^{\prime}-A_{1}^{\prime}=A_{3}-\left(A_{3}-A_{2}\right)=A_{2}<A_{3}-A_{1}=A_{2}^{\prime}$. So we may assume without loss of generality that $A_{3}-A_{1} \leq A_{2}$.

If $A_{3}-A_{1}=A_{2}$, then exactly two of $\left\{A_{1}, A_{2}, A_{3}\right\}$ are odd, so exactly two of $\left\{e^{i A_{1} \pi}, e^{i A_{2} \pi}, e^{i A_{3} \pi}\right\}$ are equal to -1 and the other is equal to 1 . Therefore,

$$
\begin{aligned}
& \inf _{\alpha \in \mathfrak{R}} F_{4}\left(0, A_{1} \alpha, A_{2} \alpha, A_{3} \alpha\right) \leq F_{4}\left(0, A_{1} \pi, A_{2} \pi, A_{3} \pi\right) \\
& \quad=\left|1+e^{i A_{1} \pi}+e^{i A_{2} \pi}+e^{i A_{3} \pi}\right|=|1+1-1-1|^{2}=0<0.566 \ldots .
\end{aligned}
$$

So we may assume $A_{3}-A_{1}<A_{2}$. Now if $A_{1}, A_{2}, A_{3}$ violate one of the inequalities (iv)-(vi) in Lemma 3, we have, by Lemma 2 , with $w=4.18$, that

$$
\inf _{\alpha \in \mathfrak{R}} F_{4}\left(0, A_{1} \alpha, A_{2} \alpha, A_{3} \alpha\right)<\left[\frac{\pi}{4.18}\right]^{2}<0.566 \ldots=\inf _{\alpha \in \mathfrak{R}} F_{4}(0,2 \alpha, 3 \alpha, 4 \alpha) .
$$


Therefore, to find $\sup _{0<A_{1}<A_{2}<A_{3}} \inf _{\alpha \in \mathfrak{R}}\left|F_{4}\left(0, A_{1} \alpha, A_{2} \alpha, A_{3} \alpha\right)\right|$, it suffices to look only at triples $\left(A_{1}, A_{2}, A_{3}\right)$ that satisfy the hypotheses of Lemma 3 . But this means we need only check $(2,3,4)$ and $(4,9,10)$. Now

$$
\begin{aligned}
\inf _{\alpha}\left|F_{4}(0,4 \alpha, 9 \alpha, 10 \alpha)\right| & \leq F_{4}\left(0,4\left(\frac{4}{10}\right), 9\left(\frac{4}{10}\right), 10\left(\frac{4}{10}\right)\right) \\
& =0.3758 \ldots<0.566 \ldots
\end{aligned}
$$

Therefore

$$
\begin{aligned}
f(A)= & \sup _{\substack{0<A_{1}<A_{2}<A_{3} \\
\operatorname{gcd}\left(A_{1}, A_{2}, A_{3}\right)=1}} \inf _{\alpha \in \mathfrak{R}}\left(F_{4}\left(0, A_{1} \alpha, A_{2} \alpha, A_{3} \alpha\right)\right)^{1 / 2} \\
= & \inf _{\alpha \in \mathfrak{R}}\left(F_{4}(0,2 \alpha, 3 \alpha, 4 \alpha)\right)^{1 / 2}=(0.5661 \ldots)^{1 / 2}=0.7524 \ldots
\end{aligned}
$$

\section{FURTHER RESULTS}

In an effort to see how fast $f(n)$ grows (or see if it is, in fact, monotonic), we explicitly computed several examples to estimate the size of $f(5)$ and $f(6)$.

First, we generated all quadruples $\left(A_{1}, A_{2}, A_{3}, A_{4}\right)$ of natural numbers with $\operatorname{gcd}\left(A_{1}, A_{2}, A_{3}, A_{4}\right)=1$ and $0<A_{1}<A_{2}<A_{3}<A_{4} \leq 30$. For each quadruple, we computed the values of

$$
F_{5}\left(0, A_{1} \alpha, A_{2} \alpha, A_{3} \alpha, A_{4} \alpha\right) \text { for } \alpha=0,0.01,0.02, \ldots, 3.15
$$

and saved the smallest value. The largest of these came from the quadruple $(1,2,6,9)$. The minimum value of $F_{5}(0, \alpha, 2 \alpha, 6 \alpha, 9 \alpha)$ apparently occurs when $\alpha=\pi$ and gives the surprising value

$$
\begin{aligned}
F_{5}(0, \pi, 2 \pi, 6 \pi, 9 \pi) & =\left|1+e^{i \pi}+e^{i 2 \pi}+e^{i 6 \pi}+e^{i 9 \pi}\right| 2 \\
& =|1-1+1+1-1|^{2}=1,
\end{aligned}
$$

which also appears in Table 1 of [5]. Thus $f(5) \geq 1$. We did the same for $f(6)$. We checked all tuples $0<A_{1}<A_{2}<A_{3}<A_{4}<A_{5} \leq 30$ and all values of $\alpha$ from 0 to $\pi$ in increments of 0.001 and found that

$$
\begin{aligned}
f(6) & =\sup _{0<A_{1}<\cdots<A_{5}} \inf _{\alpha \in \mathfrak{R}}\left|1+\sum_{j=1}^{5} e^{i A_{j} \alpha}\right| \\
& \geq \inf _{\alpha \in \mathfrak{R}}\left|1+e^{i 6 \alpha}+e^{i 9 \alpha}+e^{i 10 \alpha}+e^{i 17 \alpha}+e^{i 24 \alpha}\right| \approx 1.1348 \ldots,
\end{aligned}
$$

which is achieved when $\alpha \approx 2.45$.

Thus the Newman polynomials $1+z+z^{2}+z^{6}+z^{9}$ and $1+z^{6}+z^{9}+z^{10}+$ $z^{17}+z^{24}$ have only five and six terms, but yet have minimum modulus on the unit circle larger than or equal to 1. In [5] Boyd shows that $f(n)>1$ for $6 \leq n \leq 16$ and conjectures that $\log f(n) / \log n \rightarrow \alpha>0$. It seems quite likely that $f(n)$ is at least monotonic.

\section{ACKNOWLEDGMENT}

The referee is to be commended for his insightful comments and thoughtful work. 


\section{REFERENCES}

1. Paul Erdös, Some unsolved problems, Michigan Math. J. 4 (1957), 291-300.

2. J. E. Littlewood, On polynomials $\sum^{n} \pm z^{m}, \sum^{n} e^{\alpha_{m} i} z^{m}, z=e^{\theta_{i}}$, J. London Math. Soc. (2) 41 (1966), 367-376.

3. D. M. Campbell, H. R. P. Ferguson, and R. W. Forcade, Newman polynomials on $|z|=1$, Indiana Univ. Math. J. 32 (1983), 517-525.

4. C. J. Smyth, Some results on Newman polynomials, Indiana Univ. Math. J. 34 (1985), 195-200.

5. D. W. Boyd, Large Newman polynomials in Diophantine analysis (J. Loxton and A. van der Poorten, eds.), London Math Soc. Lecture Notes Ser., vol. 109, Cambridge Univ. Press, Cambridge and New York, 1986.

6. F. W. Carroll, D. Eustice, and T. Figiel, The minimum modulus of polynomials with coeffcients of modulus one, J. London Math. Soc. (2) 16 (1977), 76-82.

7. D. A. Brannan and J. G. Clunie, eds., Aspects of contemporary complex analysis, Academic Press, New York, 1980, p. 561.

8. G. H. Hardy and E. M. Wright, An introduction to the theory of numbers, Clarendon Press, Oxford, 1979.

Department of Mathematics, Rose-Hulman Institute of Technology, Terre Haute, INDIANA 47803-3999

E-mail address: goddard@nextwork.rose-hulman.edu 